\title{
The transcriptional regulator BZR1 mediates trade-off between plant innate immunity and growth
}

\author{
Rosa Lozano-Durán ${ }^{1}$, Alberto P Macho ${ }^{1}$, Freddy Boutrot ${ }^{1}$, Cécile Segonzac $^{1 \dagger}$, \\ Imre E Somssich², Cyril Zipfel ${ }^{1 *}$
}

${ }^{1}$ The Sainsbury Laboratory, Norwich, United Kingdom; ${ }^{2}$ Max Planck Institute for Plant Breeding Research, Köln, Germany

\begin{abstract}
The molecular mechanisms underlying the trade-off between plant innate immunity and steroid-mediated growth are controversial. Here, we report that activation of the transcription factor BZR1 is required and sufficient for suppression of immune signaling by brassinosteroids (BR). BZR1 induces the expression of several WRKY transcription factors that negatively control early immune responses. In addition, BZR1 associates with WRKY40 to mediate the antagonism between BR and immune signaling. We reveal that BZR1-mediated inhibition of immunity is particularly relevant when plant fast growth is required, such as during etiolation. Thus, BZR1 acts as an important regulator mediating the trade-off between growth and immunity upon integration of environmental cues. DOI: 10.7554/eLife.00983.001
\end{abstract}

*For correspondence: cyril. zipfel@tsl.ac.uk

tPresent address: Institute of Agriculture and Environment, Massey University Manawatu, Palmerston North, New Zealand

Competing interests: The authors declare that no competing interests exist.

Funding: See page 12

Received: 22 May 2013

Accepted: 18 November 2013

Published: 31 December 2013

Reviewing editor: Thorsten Nürnberger, University of

Tübingen, Germany

(c) Copyright Lozano-Durán et al. This article is distributed under the terms of the Creative Commons Attribution License, which permits unrestricted use and redistribution provided that the original author and source are credited.

\section{Introduction}

The trade-off between plant growth and immunity needs to be finely regulated to ensure proper allocation of resources in an efficient and timely manner upon effective integration of environmental cues (Pieterse et al., 2012). A key aspect of plant immunity is the perception of pathogen-associated molecular patterns (PAMPs) by surface-localized pattern-recognition receptors (PRRs), leading to PAMP-triggered immunity (PTI) (Dodds and Rathjen, 2010). PRRs of the leucine-rich repeat receptor kinases (LRR-RKs) class rely on the regulatory LRR-RK BAK1 (BRASSINOSTEROID INSENSITIVE 1-ASSOCIATED KINASE 1) for signaling (Monaghan and Zipfel, 2012); that is the case of FLS2 (FLAGELLIN SENSITIVE 2) and EFR (EF-TU RECEPTOR), which perceive bacterial flagellin (or the active peptide flg22) and EF-Tu (or the active peptide elf18) respectively. BAK1 also interacts with the LRR-RK BRI1 (BRASSINOSTEROID INSENSITIVE 1), the main receptor for the growth-promoting steroid hormones brassinosteroids (BR), and is a positive regulator of BR-mediated growth (Kim and Wang, 2010). Hence, a crosstalk between the BR- and PAMP-triggered signaling pathways resulting from competition for BAK1 was hypothesized. While a unidirectional antagonism between BR and PTI signaling has been recently described in Arabidopsis (Albrecht et al., 2012; Belkhadir et al., 2012), the exact underlying mechanisms are still controversial. Activation of the BR signaling pathway via either transgenic overexpression of BRI1 or the BR biosynthetic gene DWF4 or expression of the activated BRI1 allele BRI1 sud suppresses PTI outputs (Belkhadir et al., 2012). One such output, the PAMP-triggered callose deposition, could be restored by over-expression of BAK1-HA, suggesting that BAK1 is a limiting factor (Belkhadir et al., 2012). However, exogenous BR treatment of wild-type plants does not affect the FLS2-BAK1 complex formation upon FLS2 activation, while it results in decreased PTI responses (Albrecht et al., 2012).

\section{Results and discussion}

In order to clarify the role of BAK1 in the BR-PTI crosstalk, we investigated FLS2-BAK1 complex formation in the transgenic Arabidopsis lines overexpressing BRI1 or DWF4 or expressing BRI1 sud (Belkhadir et al., 
eLife digest Like all organisms, plants must perform a careful balancing act with their resources. Investing in the growth of new roots or leaves can allow a plant to better exploit its environmentbut it must not be at the expense of leaving the plant vulnerable to attack by pests and pathogens. As such, there is an obvious trade-off between allocating resources to growth or defense against disease. This trade-off must be finely balanced, and must also be responsive to different cues in the environment that would favor either growth or defense.

The plant's immune system is able to detect invading microbes, and trigger a defensive response against them. At the surface of plant cells, proteins called pattern recognition receptors are able to recognize specific molecules that are the tell-tale signs of microbes and pathogens-such as the proteins in the molecular tails that bacteria use to move around.

For many pattern recognition receptors, signaling that they have recognized a potential invading microbe requires the actions of a co-receptor called BAK1. Interestingly, BAK1 also interacts with the receptor that identifies brassinosteroids-hormones that stimulate plant growth. Since growth and a functioning immune system are both reliant on BAK1, it was hypothesized that competition for this co-receptor could have a role in the trade-off between the two processes in plants. However, this explanation was controversial and the mechanisms underlying the trade-off still required clarification.

Now, Lozano-Durán et al. have debunked the idea that competition for BAK1 is directly responsible for the trade-off between growth and immunity. By examining how BAK1 interacts with immune receptors in the plant model species Arabidopsis thaliana, the trade-off was actually shown to be independent of BAK1. Instead, it was discovered that activation of a protein, called BZR1, reprogramed gene expression to 'switch off' immune signaling in response to brassinosteroids.

Lozano-Durán et al. also show that BZR1 allows the balance of the trade-off between growth and immunity to be shifted in response to cues from the environment. The suppression of the immune system by BZR1 was particularly pronounced when the conditions required fast plant growth-for example, when they mimicked the conditions experienced by seedlings before they emerge from the soil, and must grow swiftly to reach the light before they starve.

DOI: $10.7554 /$ Life.00983.002

2012). Upon treatment with flg22, FLS2 associated normally with BAK1 in these transgenic plants, and neither FLS2 nor BAK1 accumulation was altered (Figure 1-figure supplement 1A). Moreover, these plants displayed a weaker reactive oxygen species (ROS) burst in response to chitin (Figure 1-figure supplement 1B), whose signaling pathway is BAK1-independent (Shan et al., 2008; Ranf et al., 2011). This result is consistent with the previous finding that exogenous BR treatment can also inhibit the chitin-induced ROS burst (Albrecht et al., 2012). BAK1-HA is not fully functional in BR signaling and exerts a dominant-negative effect on the endogenous BAK1 (Figure 1-figure supplement 1C), which may explain that introduction of the BAK1-HA transgene can override the suppression of immunity triggered by overexpression of BRI1 (Belkhadir et al., 2012); BAK1-HA does not exert such a dominant negative effect, however, on PTI signaling (Figure 1-figure supplement 1D). Taken together, these results indicate that the BR-mediated suppression of $\mathrm{PTI}$ is triggered independently of a competition between BRI1 and PRRs for BAK1.

We sought to determine at which level of the BR signaling pathway the antagonism initiates. After BR perception by BRI1 and activation of the BRI1-BAK1 complex, the BR signal transduction cascade includes inactivation of BIN2 (BR INSENSITIVE 2) and BIN2-like kinases, a family of GSK3-like kinases acting as negative regulators of the pathway (Vert and Chory, 2006). This leads to dephosphorylation of BZR1 (BRASSINAZOLE RESISTANT 1) and BES1/BZR2 (BRI1-EMS-SUPPRESSOR 1/BRASSINAZOLE RESISTANT 2), two bHLH transcription factors acting as major regulators of BR-induced transcriptional changes, which then become active (Wang et al., 2002; Yin et al., 2002). Treatment with the chemicals $\mathrm{LiCl}$ and bikinin, which inhibit GSK3-like kinases (De Rybel et al., 2009; Yan et al., 2009), resulted in impaired flg22-triggered ROS burst (Figure 1A,B), as observed upon genetic or ligand-induced activation of the BR pathway. Furthermore, a triple mutant in BIN2 and the two closest related GSK3-like kinases, BIL1 (BIN2-LIKE 1) and BIL2 (triple GSK3 mutant; Vert and Chory, 2006), shows a similar impairment in response to either flg22 or chitin (Figure 1C). Interestingly, in spite of regulating MAPKs involved in 


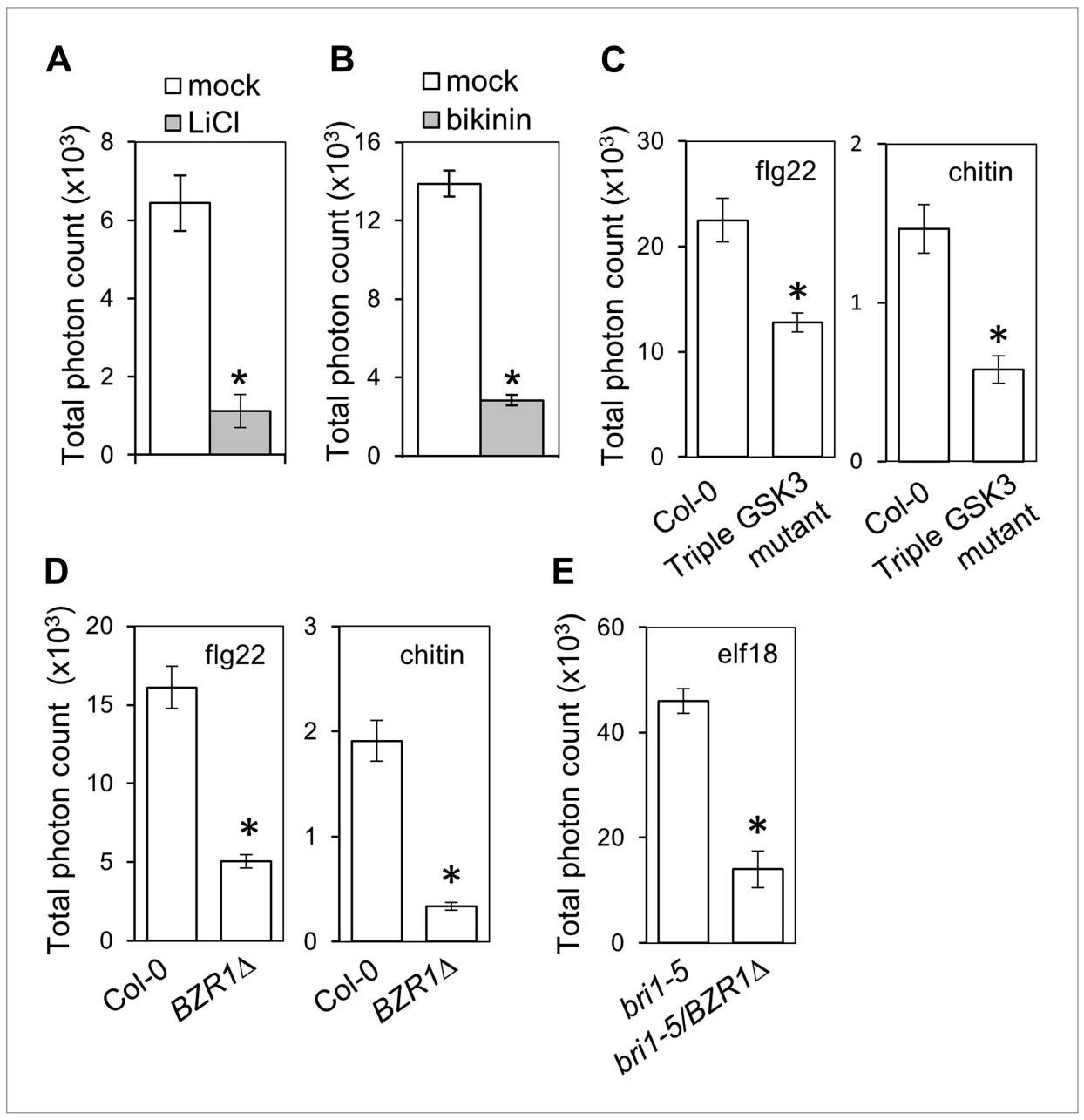

Figure 1. Activation of BZR1 is sufficient to inhibit the PAMP-triggered ROS burst. (A) and (B) Flg22-triggered ROS burst after $\mathrm{LiCl}(\mathbf{A})$ or bikinin (B) treatment. Leaf discs were pre-treated with a $10 \mathrm{mM} \mathrm{LiCl}$ solution for $5 \mathrm{hr}$ or with a $50 \mu \mathrm{M}$ bikinin solution for $16 \mathrm{hr}$. (C) Flg22- or chitin-triggered ROS burst in Col-0 and the triple GSK3 mutant plants. (D) Flg22- or chitin-induced ROS burst in Col-0 and BZR1 $\Delta$ plants. (E) Elf18-triggered ROS burst in bri1-5 and bri1-5/ BZR1 $\triangle$ plants. In all cases, bars represent SE of $n=28$ rosette leaf discs. Asterisks indicate a statistically significant difference compared to the corresponding control (mock treatment $[\mathbf{A}$ and $\mathbf{B}]$, Col-0 [C and $\mathbf{D}]$ or bri1-5 [E]), according to a Student's $t$-test $(p<0.05)$. Leaf discs of four- to five-week-old Arabidopsis plants were used in these assays. Flg22 and elf18 were used at a concentration of $50 \mathrm{nM}$; chitin was used at a concentration of $1 \mathrm{mg} / \mathrm{ml}$. Total photon counts were integrated between minutes two and 40 after PAMP treatment. All experiments were repeated at least three times with similar results.

DOI: 10.7554/eLife.00983.003

The following figure supplements are available for figure 1:

Figure supplement 1. The BR-mediated suppression of PTI can be triggered independently of a competition for BAK1. DOI: 10.7554/eLife.00983.004

Figure supplement 2. PAMP-triggered MAPK activation is not impaired upon activation of BR signaling DOI: 10.7554/eLife.00983.005

Figure supplement 3. Activation of BZR1, but not BES1, is sufficient to inhibit the PAMP-triggered ROS burst. DOI: 10.7554/eLife.00983.006

stomata development (Kim et al., 2012; Khan et al., 2013), neither BR treatment nor loss of function of BIN2 affect flg22-triggered MAPK activation (Figure 1-figure supplement 2), contrary to what has been recently suggested (Choudhary et al., 2012; Zhu et al., 2013). These results indicate that the BR-PTI crosstalk occurs downstream of BIN2.

Transgenic expression of two different constitutively active versions of BZR1, BZR1 (Gampala et al., 2007) and BZR15173A (Ryu et al., 2007), results in impaired flg22- or chitin-triggered ROS burst 
(Figure 1D, Figure 1-figure supplement 3A). Consistent with previous results (Albrecht et al., 2012; Figure 1-figure supplements $1 A$ and 2), plants expressing BZR1 $\triangle$ or BZR1S173A display normal FLS2-BAK1 complex formation and MAPK activation upon flg22 treatment (Figure 2A, B, Figure 2figure supplement $1 \mathrm{~A}, B$ ), but are impaired in PAMP-triggered marker gene expression, seedling growth inhibition (SGI) (Figure 2C-E) and induced resistance to P. syringae pv. tomato (Pto) DC3000

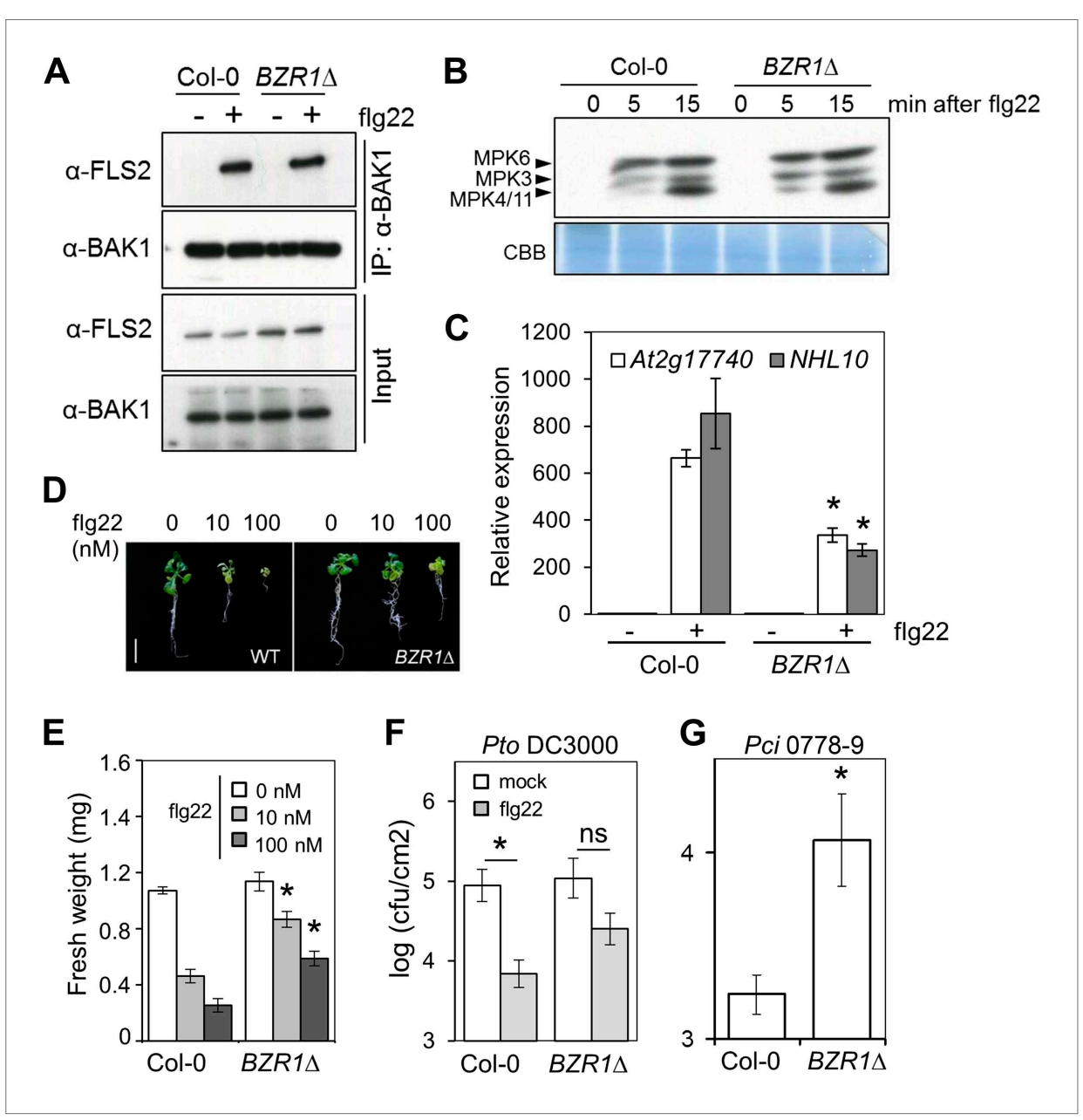

Figure 2. Activation of BZR1 results in the suppression of specific PTI outputs. (A) Co-immunoprecipitation (Co-IP) of BAK1 and FLS2 in Col-0 and BZR1 $\triangle$ seedlings after 10 min mock (-) or $1 \mu \mathrm{M} \mathrm{flg22} \mathrm{(+)} \mathrm{treatment.} \mathrm{Proteins} \mathrm{were}$ separated in a $10 \%$ acrylamide gel and transferred to PVDF membranes. Membranes were blotted with anti-FLS2 or anti-BAK1 antibodies. (B) MAPK activation in Col-0 and BZR1 $\Delta$ seedlings upon $1 \mu \mathrm{M}$ flg22 treatment. Proteins were separated in a $10 \%$ acrylamide gel and transferred to PVDF membranes. Membranes were blotted with phospho-p44/42 MAPK (Erk1/2; Thr202/Tyr204) rabbit monoclonal antibodies. CBB: Coomassie brilliant blue. (C) Marker gene (At2g17700 and NHL10) expression in Col-0 and BZR1 $\Delta$ seedlings after $1 \mathrm{hr}$ mock (-) or $1 \mu \mathrm{M}$ flg22 (+) treatment, as determined by qPCR. Bars represent SE of $n=3$. (D) and (E) Seedling growth inhibition of 10-day-old Col-0 or BZR1 $\triangle$ seedlings induced by increasing concentrations of flg22, as indicated. Scale bar (D), $1 \mathrm{~cm}$. Bars (E) represent $\mathrm{SE}$ of $8 \leq \mathrm{n} \leq 16$. (F) Flg22-induced resistance to $P$. syringae pv. tomato DC3000 in Col-0 and BZR1 $\Delta$ plants. Plants were pre-treated with $1 \mu \mathrm{M}$ flg22 or water $24 \mathrm{hr}$ prior to bacterial infiltration. Bars represent $\mathrm{SE}$ of $\mathrm{n}=4$. This experiment was repeated seven times with similar results. (G) Susceptibility of Col-0 and BZR1 $\triangle$ plants to $P$. syringae pv. cilantro 0788-9. Bars represent $\mathrm{SE}$ of $\mathrm{n}=4$. Asterisks indicate a statistically significant difference compared to Col-0 according to a Student's $t$-test $(p<0.05)$; $n s=$ not significant. All experiments were repeated at least twice with similar results unless otherwise stated.

DOI: 10.7554/eLife.00983.007

The following figure supplements are available for figure 2:

Figure supplement 1 . Expression of the constitutively active BZR1 ${ }^{5173 A}$ results in the suppression of specific PTI outputs. DOI: 10.7554/eLife.00983.008 
(Figure 2F, Figure 2-figure supplement 1C), and are more susceptible to the non-host strain Pseudomonas syringae pv. cilantro (Pci) 0788 -9 (Lewis et al., 2010) (Figure 2G). Notably, transgenic expression of a constitutively active form of BES1, BES1 ${ }^{\mathrm{S} 171 \mathrm{~A}}$ (Gampala et al., 2007), does not impact the flg22-triggered ROS burst (Figure 1-figure supplement 3B). We then tested if activation of BZR1 is sufficient to inhibit PTI signaling. Induction of BR signaling by bikinin treatment still represses elf18induced ROS burst in the BRI1 mutant bri1-5 (we used elf18 in this experiment because bri1-5 is in the Ws-2 background, which is a natural fls2 mutant) (Figure 1-figure supplement 3C). bri1-5/BZR1 $\triangle$ plants (Gampala et al., 2007) still exhibited reduced PAMP-triggered ROS burst (Figure 1E), and treatment with the BR biosynthetic inhibitor brassinazole (BRZ) did not affect the BZR1 $\Delta$ effect (Figure 1-figure supplement 3D). Interestingly, BRZ treatment of wild-type Col-0 plants results in increased ROS production (Figure 1-figure supplement 3D), which is consistent with the fact that BR inhibits PTI responses and suggests that endogenous concentrations of the hormone exert this effect. These results demonstrate that activation of BZR1 affects PTI signaling independently of BR perception or synthesis.

To understand how BZR1 mediates the BR-PTI crosstalk, we performed meta-analysis of microarray and ChIP-chip data containing BR-regulated and BZR1 or BES1 target genes (Sun et al., 2010; Yu et al., 2011). Functional enrichment of BR-regulated genes unveiled a statistically significant overrepresentation of defense-related GO terms of the Biological Process ontology (Table 1), indicating that $B R$ signaling regulates the expression of defense-related genes. Independent analysis of BR-regulated BZR1 or BES1 targets confirmed BZR1 as the main transcription factor involved in the regulation of defense gene expression (Table 1). Two out of four over-represented GO terms of the Molecular Function ontology among the BR-regulated BZR1 targets are transcription factor and transcription repressor activity (Table 2). Interestingly, several defense-related GO terms are also over-represented in the subset of BR-regulated BZR1-targeted transcription factors (Table 3), pointing at a BZR1-mediated secondary transcriptional wave of defense-related genes.

To identify BZR1-regulated transcription factors with a prominent role in defense, we performed promoter enrichment analysis on the subset of defense-related BR-regulated genes, and found the W-box motif as the only significantly over-represented motif (Table 4). The W-box motif is the binding site for the WRKY family of transcription factors (Rushton et al., 2010), and several members of this family are BR-regulated BZR1-targets (Table 5). We hypothesized that WRKYs that are BR-induced and BZR1 targets may be involved in PTI signaling. Notably, wrky11, wrky15, wrky18 and wrky70 mutants displayed enhanced PAMP-triggered ROS (Figure 3A), suggesting that these transcription factors

Table 1. Defense-related Gene Ontology terms (Biological Process ontology) over-represented among all BR-regulated genes, BR-regulated BZR1 targets and BR-regulated BES1 targets

\begin{tabular}{|c|c|c|c|}
\hline Defense-related GO term & Observed frequency (\%) & Expected frequency (\%) & p-value \\
\hline \multicolumn{4}{|l|}{ BR-Regulated genes } \\
\hline response to bacterium & 2.2 & 1 & $3.31 \times 10^{-08}$ \\
\hline defense response to bacterium & 1.9 & 0.8 & $3.31 \times 10^{-08}$ \\
\hline response to chitin & 1.4 & 0.5 & $1.78 \times 10^{-07}$ \\
\hline defense response & 4.7 & 3 & $3.32 \times 10^{-07}$ \\
\hline response to fungus & 1.5 & 0.7 & $3.4 \times 10^{-06}$ \\
\hline response to nematode & 0.7 & 0.2 & 0.000532 \\
\hline defense response to fungus & 1 & 0.5 & 0.0035 \\
\hline \multicolumn{4}{|l|}{ BR-regulated BZR1 targets } \\
\hline response to chitin & 2.6 & 0.5 & $9.13 \times 10^{-13}$ \\
\hline response to bacterium & 2.3 & 1 & 0.00112 \\
\hline defense response to bacterium & 1.9 & 0.8 & 0.00154 \\
\hline response to fungus & 1.6 & 0.7 & 0.00495 \\
\hline \multicolumn{4}{|l|}{ BR-regulated BES1 targets } \\
\hline response to chitin & 2.4 & 0.5 & 0.00439 \\
\hline
\end{tabular}

DOI: 10.7554/eLife.00983.009 
Table 2. Gene Ontology terms (Molecular Function ontology) over-represented among all BR-regulated BZR1 targets

\begin{tabular}{lccc}
$\begin{array}{l}\text { Over-represented GO term } \\
\text { BR-regulated BZR1 targets }\end{array}$ & Observed frequency (\%) & Expected frequency (\%) & p value \\
\hline $\begin{array}{l}\text { nucleic acid binding } \\
\text { transcription factor activity }\end{array}$ & 14.8 & 10.2 & 0.000223 \\
\hline transferase activity & 21.6 & 16.8 & 0.00333 \\
\hline kinase activity & 11.6 & 8.1 & 0.00702 \\
\hline transcription repressor activity & 1.1 & 0.3 & 0.01
\end{tabular}

DOI: 10.7554/eLife.00983.010

act as negative regulators of early PTI signaling. This is in accordance with their role as negative regulators of immunity (Figure 3-figure supplement 1A; Journot-Catalino et al., 2006). Therefore, the BZR1-mediated inhibition of PTI might be partially explained by the up-regulation of genes encoding WRKY transcription factors that negatively control the expression of genes involved in early PTI signaling.

One of the WRKY genes targeted by BZR1 is WRKY40 (Sun et al., 2010). Interestingly, all described targets of WRKY40 (Pandey et al., 2010) are also targets of BZR1 (Sun et al., 2010) (Table 6). The over-representation of the W-box motif among BZR1 targets (Table 7) suggests that BZR1 may interact with WRKY transcription factors (such as WRKY40) to cooperatively regulate transcription. WRKY40 has been described as a negative regulator of defense against biotrophic pathogens and insects ( $X u$ et al., 2006; Pandey et al., 2010; Brotman et al., 2013; Schon et al., 2013; Schweizer et al., 2013). In agreement with this, we found that a null wrky40 mutant is more resistant to Pto DC 3000 (Figure 3-figure supplement 1B). Strikingly, wrky40 plants are partially impaired in the BR-mediated suppression of PAMP-triggered ROS (Figure 3B), suggesting that WRKY40 may act coordinately with BZR1 to suppress immunity. Indeed, we found that BZR1 associates with WRKY40, but not WRKY6, in co-immunoprecipitation experiments when transiently co-expressed in Nicotiana benthamiana leaves (Figure 3C) or Arabidopsis protoplasts (Figure 3D). Collectively, these results indicate that BZR1 and WRKY40 form a protein complex that may participate in the transcriptional inhibition of PTI signaling.

BZR1, together with DELLAs and PIF4, is part of a core transcription module that integrates hormonal (gibberellin [GA] and BR) and environmental (light) signals (Gallego-Bartolome et al., 2012; Li et al., 2012; Oh et al., 2012; Bai et al., 2012b). In the dark, BZR1 is activated by endogenous BR and GA to promote growth, partially through the synergistic interaction with PIF4 (Jaillais and Vert, 2012).

Table 3. Defense-related Gene Ontology terms (Biological Process ontology) over-represented among the BZR1-target BR-regulated transcription factors

\begin{tabular}{llll} 
Defense-related GO Term & $\begin{array}{l}\text { Observed } \\
\text { frequency (\%) }\end{array}$ & $\begin{array}{l}\text { Expected } \\
\text { frequency (\%) }\end{array}$ & p value \\
\hline BZR1-target BR-regulated TFs & 16.6 & 0.5 & $1.36 \times 10^{-26}$ \\
\hline $\begin{array}{l}\text { response to chitin } \\
\text { defense response to bacterium }\end{array}$ & 7.6 & 0.8 & $4.71 \times 10^{-07}$ \\
\hline $\begin{array}{l}\text { response to bacterium } \\
\text { regulation of defense response } \\
\text { to virus by host }\end{array}$ & 1.4 & 1 & $4.51 \times 10^{-06}$ \\
\hline $\begin{array}{l}\text { regulation of immune effector process } \\
\text { regulation of defense response to virus }\end{array}$ & 1.4 & 0 & 0.000964 \\
\hline $\begin{array}{l}\text { regulation of defense response } \\
\text { defense response }\end{array}$ & 2.8 & 0 & 0.00151 \\
\hline response to fungus & 8.3 & 0 & 0.00151 \\
\hline defense response to fungus & 3.4 & 0.3 & 0.00484 \\
\hline
\end{tabular}

DOI: 10.7554/eLife.00983.011 
Table 4. Over-represented cis-acting promoter elements among the defense-related BRregulated genes according to Athena (http:// www.bioinformatics2.wsu.edu/cgi-bin/Athena/ cgi/home.pl)

\begin{tabular}{lcc} 
Enriched TF site & $\%$ promoters & p value \\
\hline Defense-related BR-regulated genes & \\
\hline W-box & 72.4 & $<10^{-6}$ \\
\hline
\end{tabular}

DOI: 10.7554/eLife.00983.012 However, activation of BES1 in the BES1 ${ }^{\text {S171A }}$ line did not impact SGI (Figure 4-figure supplement 3A). Consistent with the previous results, exogenous BR treatment suppressed SGI in both light and dark (Figure 4C, Figure 4-figure supplement 2B,C). While treatment with GA alone did not have a dramatic effect on SGI, co-treatment with $B L$ and $G A$ resulted in an enhancement of the BR-mediated suppression of SGI (Figure 4C, Figure 4-figure supplement 2B), indicating an additive effect of these two hormones when applied together. Moreover, treatment with the GA synthesis inhibitors paclobutrazol (PAC) or uniconazole (Uni) abolished the effect of BL on SGI (Figure 4-figure supplement $1 A, B$ and Figure 4-figure supplement $2 B, C)$, and this effect was reduced in the GA biosynthetic mutant ga1-3 (Figure 4-figure supplement 1C). Taken together, these results demonstrate that BR suppress at least one PTI output, SGI, in the dark in a GA-dependent manner, most likely through activation of BZR1. Notably, although the wrky40 mutant undergoes etiolation normally (Figure 4-figure supplement 2D), it shows a diminished suppression of SGI in the dark (Figure 4D, Figure 4-figure supplement 2D), supporting the idea that WRKY40 is required for the BZR1-mediated inhibition of PTI.

Previously, a unidirectional negative crosstalk between the growth-promoting hormone BR and PTI had been described (Albrecht et al., 2012; Belkhadir et al., 2012). In this work, we show that activation of one of two major BR-activated transcription factors, BZR1, is sufficient to suppress PTI, measured as PAMP-triggered ROS production, PAMP-triggered gene expression, SGI or induced resistance (Figures 1 and 2, Figure 1-figure supplement 3, Figure 2-figure supplement 1). Of note, another PTI output, MAPK activation, is not affected by activation of the BR pathway

Table 5. BR-regulated BZR1-target WRKY genes

\begin{tabular}{ll} 
AGI number & WRKY TF \\
\hline BR-Induced BZR1 targets & \\
\hline AT4G31800 & WRKY18 \\
\hline AT4G31550 & WRKY11 \\
\hline AT4G23810 & WRKY53 \\
\hline AT3G56400 & WRKY70 \\
\hline AT5G49520 & WRKY48 \\
\hline AT5G52830 & WRKY27 \\
\hline AT1G69310 & WRKY57 \\
\hline AT2G23320 & WRKY15 \\
\hline BR-repressed BZR1 targets & (Yu et al., 2011) \\
\hline AT4G01250 & \\
\hline AT1G80840 & WRKY22 \\
\hline AT2G24570 & WRKY40 \\
\hline AT2G23320 & WRKY17 \\
\hline AT2G30590 & WRKY15 \\
\hline (Sun et al., 2010) \\
\hline
\end{tabular}

DOI: 10.7554/eLife.00983.013
(Figure 2B, Figure 1-figure supplement 2, Figure 2-figure supplement 1B). BR treatment results in BZR1-dependent changes in the expression of defense-related genes, among which several members of the WRKY family of transcription factors can be found. Because the promoters of BR-regulated defense-related genes are enriched in the W-box motif (Table 4), BZR1targeted WRKY transcription factors could be responsible for a secondary wave of transcription, ultimately leading to the suppression of PTI. In agreement with this idea, a subset of WRKYs induced by BR (WRKY11, WRKY15 and WRKY18) (Figure $3 \mathrm{~A}$ ) act as negative regulators of PAMPtriggered ROS, potentially by controlling the steady-state expression of genes encoding components required for this response. The overrepresentation of the $\mathrm{W}$-box motif among the BZR1 targets (Table 7) raises the possibility that, additionally, WRKY transcription factors could also act together with BZR1 to cooperatively regulate gene expression. We found that WRKY40 associates with BZR1 directly or indirectly in planta 


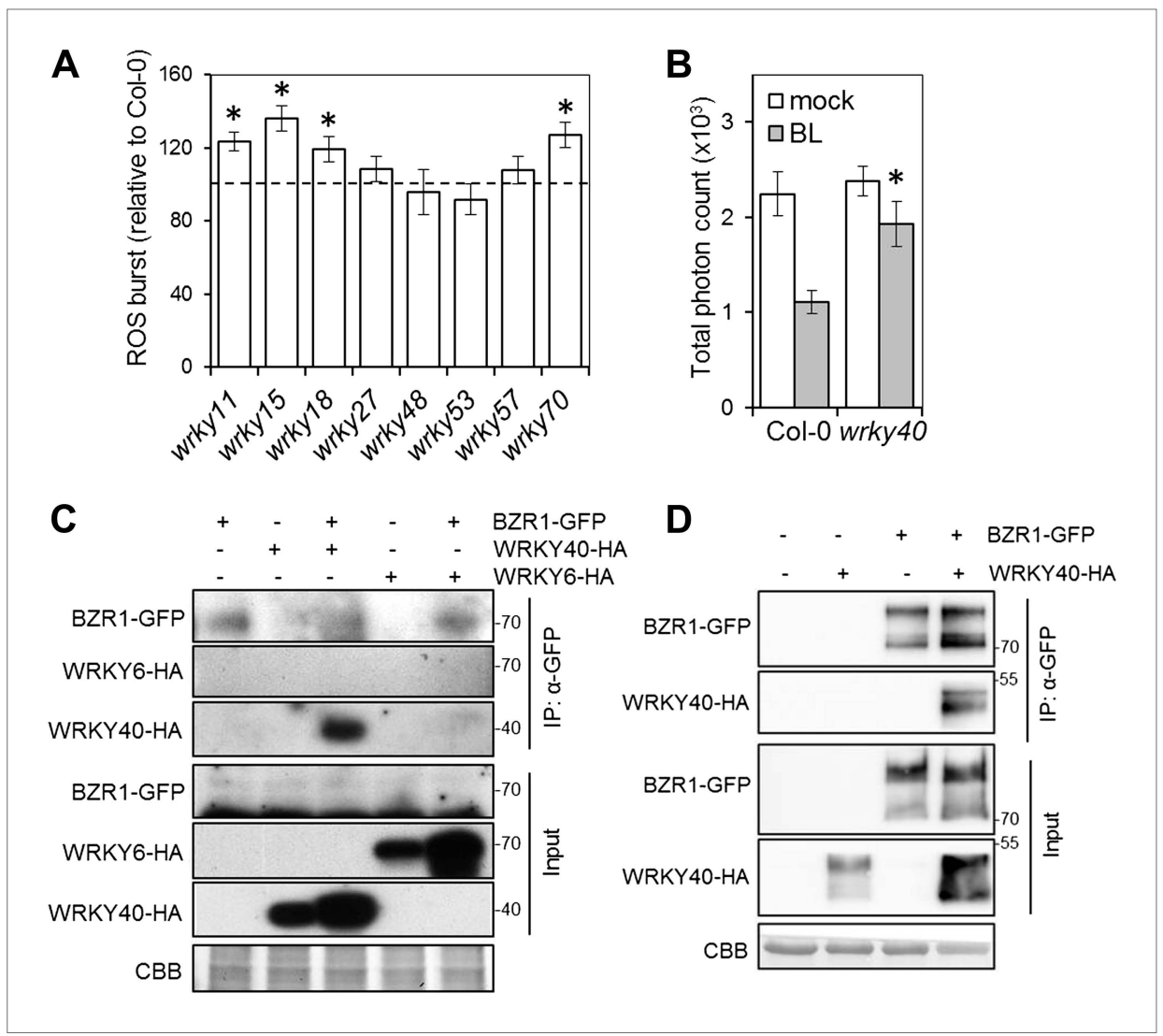

Figure 3. WRKY transcription factors play a dual role on the BR-mediated regulation of PTI signaling. (A) Flg22triggered ROS burst in mutants in each BR-induced BZR1-targeted WRKY. Leaf discs of four- to five-week-old Arabidopsis plants were used in these assays. Flg22 was used at a concentration of $50 \mathrm{nM}$. Total photon counts were integrated between minutes two and 40 after PAMP treatment. Bars represent SE of $n=28$. Asterisks indicate a statistically significant difference compared to Col-0 according to a Student's $t$-test $(p<0.05)$. (B) Flg22-triggered ROS burst in epiBL (BL)- or mock- pre-treated wrky40 mutant or wild-type plants. Leaf discs of four- to five-week-old plants were pre-treated with a $1 \mu \mathrm{M} \mathrm{BL}$ solution or mock solution for $8 \mathrm{hr}$. Flg22 was used at a concentration of $50 \mathrm{nM}$. Total photon counts were integrated between minutes two and 40 after PAMP treatment. Bars represent SE of $n=21$. Asterisks indicate a statistically significant difference compared to Col-0 according to a Student's t-test $(p<0.05)$. (C) Co-IP of BZR1-GFP transiently expressed in N. benthamiana, alone or together with WRKY40-HA or WRKY6-HA. BZR1-GFP was immunoprecipitated with an anti-GFP antibody. Immuniprecipitated or total proteins were separated in a 10\% acrylamide gel and transferred to PVDF membranes. Membranes were blotted with anti-HA or anti-GFP antibodies. CBB: Coomassie brilliant blue. (D) Co-IP of BZR1-GFP transiently expressed in Arabidopsis protoplasts, alone or together with WRKY40-HA. BZR1-GFP was immunoprecipitated with an anti-GFP antibody. Immuniprecipitated or total proteins were separated in a 10\% acrylamide gel and transferred to PVDF membranes. Membranes were blotted with anti-HA or anti-GFP antibodies. CBB: Coomassie brilliant blue. All experiments were repeated at least twice with similar results.

DOI: 10.7554/eLife.00983.014

The following figure supplements are available for figure 3:

Figure supplement 1. Mutants in WRKY11, WRKY15, WRKY18 and WRKY40 are more resistant to Pto DC3000. DOI: 10.7554/eLife.00983.015

(Figure 3C,D); in the absence of WRKY40, the BR-mediated suppression of PAMP-triggered ROS burst is partially impaired (Figure 3B). Therefore, WRKYs may play a dual role in the BZR1-mediated suppression of defense, as both co- and secondary regulators of defense gene expression. Given that the loss of BR-mediated suppression of PAMP-triggered ROS burst in the wrky40 mutant is only partial, BZR1 may interact with other members of the WRKY family, such as WRKY18 or WRKY60, to repress immunity.

Furthermore, we recently described that the bHLH transcription factor HBI1, which is a BRZ1 target (Sun et al., 2010; Bai et al., 2012a), negatively regulates PTI (Malinovsky et al., under revision). All together, these results illustrate that BZR1 controls the expression of transcription factors (e.g. WRKY11, 
Table 6. Overlap between the targets of WRKY40 and BZR1

\begin{tabular}{ll}
$\begin{array}{l}\text { Known targets of WRKY40 } \\
\text { (Pandey et al., 2010) }\end{array}$ & $\begin{array}{l}\text { Targets of BZR1 } \\
\text { (Sun et al., 2010) }\end{array}$ \\
\hline $\begin{array}{l}\text { Confirmed by ChIP } \\
\text { EDS1 }\end{array}$ & Yes \\
\hline RRTF1 & Yes \\
\hline JAZ8 & Yes
\end{tabular}

Putative (according to expression analyses)

\begin{tabular}{ll}
\hline LOX2 & Yes \\
\hline AOS & Yes \\
\hline JAZ7 & Yes \\
\hline JAZ10 & Yes \\
\hline
\end{tabular}

DOI: 10.7554/eLife.00983.016

Table 7. Representation of the W-box motif among the BR-regulated BZR1 targets according to Athena (http://www.bioinformatics2.wsu.edu/ cgi-bin/Athena/cgi/home.pl)

\begin{tabular}{lll} 
BZR1 targets & $\begin{array}{l}\% \text { of promoters } \\
\text { with W-box motif(s) }\end{array}$ & p value \\
\hline BR-induced & 66 & $<10^{-10}$ \\
\hline BR-repressed & 72 & $<10^{-4}$ \\
\hline
\end{tabular}

DOI: 10.7554/eLife.00983.017
WRKY15, WRKY18 and HBI1), which themselves might control the expression of PTI components (see model in Figure 4E) whose identities remain to be identified.

Plants need to finely regulate allocation of resources upon integration of environmental cues, both biotic and abiotic, in order to rapidly and readily adapt to changing conditions and ensure survival in a cost-efficient manner. Dark conditions impose an energetic limitation due to lack of photo-assimilates; in this situation, the restoration of normal photosynthesis by reaching light is an essential requirement to guarantee perpetuation, and as such must be given priority (Casal, 2013). We hypothesize that when plants face conditions that require rapid growth, such as when germinating in soil or when under a canopy, limited resources are invested in this developmental process at the expense of immunity in what must be a quantitative choice. Indeed, we show that etiolated seedlings do not arrest their growth in response to PAMPs as light-grown seedlings do, as measured by total fresh weight (Figure 4A-D). In addition, BR signaling, acting cooperatively with GA signaling, is required for the dark-induced suppression of this PTI response (Figure 4C, Figure 4-figure supplement 1), and activation of BZR1 is sufficient to exert this effect regardless of light conditions (Figure 4B). Although seedlings were used in these experiments due to technical reasons, BR also regulate growth at later developmental stages, so this phenom-

enon may be more general. Based on these findings, we propose a model in which BZR1 regulates the expression of defense genes, assisted by WRKY40 (and potentially other WRKYs), ultimately resulting in a quantitative suppression of immunity (Figure 4E). Because the activation status of BZR1 depends on BR, GA and light signaling, BZR1 would act as a molecular integrator of these inputs to effectively regulate the trade-off between growth and immunity.

\section{Materials and methods}

\section{Plant materials and growth conditions}

Col-0 plants were used as control. The transgenic lines BZR1A, bri1-5/BZR1 $\Delta$ and BES1 ${ }^{\text {S171A }}$ (Gampala et al., 2007), BZR1 ${ }^{\text {S173A }}$ and BZR1-CFP (Ryu et alo, 2007), 35S:BRI1-cit, BRI1p:BRI1 sud-cit, 35S:DWF4 and BAK1-HA (Belkhadir et al., 2012) are published. The mutant lines Triple GSK3 mutant (Vert and Chory, 2006), bri1-5 (Noguchi et al., 1999), bri1-301 (Xu et al., 2008), bin2-1 (Peng et al., 2008), wrky11 (Journot-Catalino et al., 2006), wrky18, wrky53 and wrky70 (Wang et al., 2006) wrky27 (Mukhtar et al., 2008), wrky40 (Pandey et al., 2010) and ga1-3 (Navarro et al., 2008) are published. wrky15 mutant was identified in the ZIGIA population (Wisman et al., 1998a, 1998b); wrky48 and wrky57 are from the SALK collection (Alonso et al., 2003).

Arabidopsis plants and seedlings were grown as described in Albrecht et al. (2012).

\section{Chemicals}

Flg22 and elf18 peptides were purchased from Peptron, and chitin oligosaccharide from Yaizu Suisankagaku. epiBL was purchased from Xiamen Topusing Chemical. LiCl, bikinin, brassinazole and GA were purchased from Sigma (St Louis, MO, USA). Paclobutrazol was purchased from Duchefa (Haarlem, NL). Uniconazole was purchased from Sigma. 
A

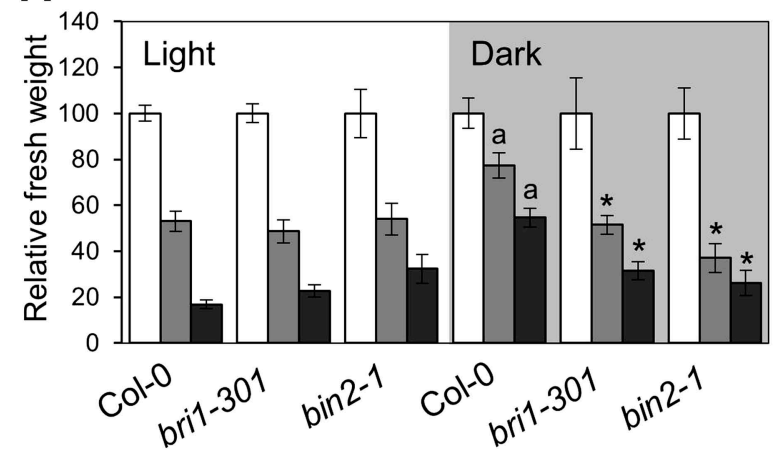

C

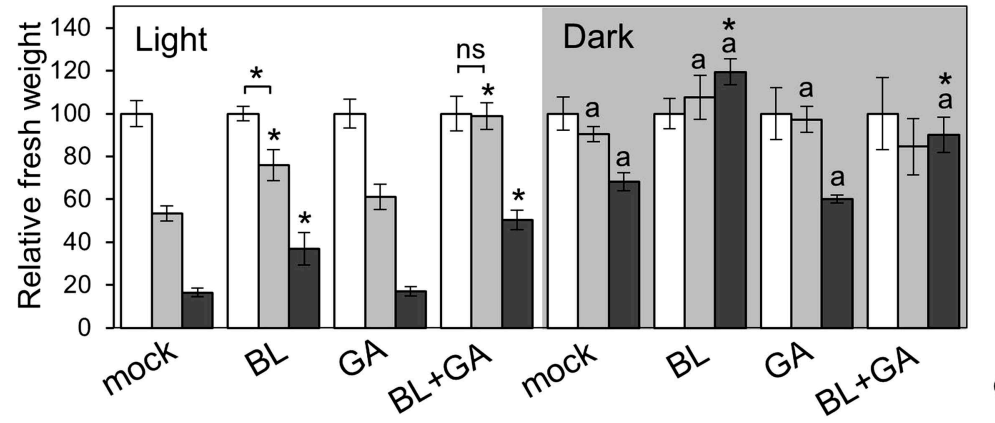

B

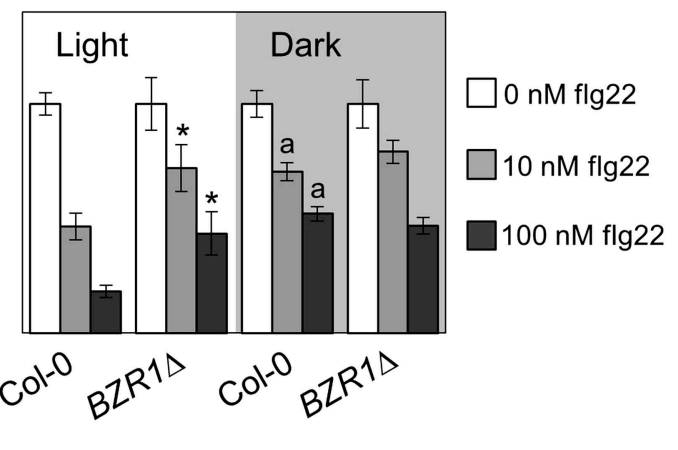

D

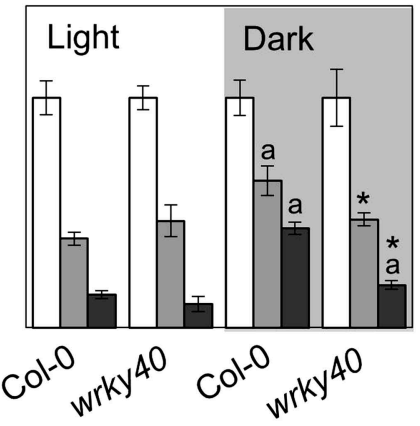

E

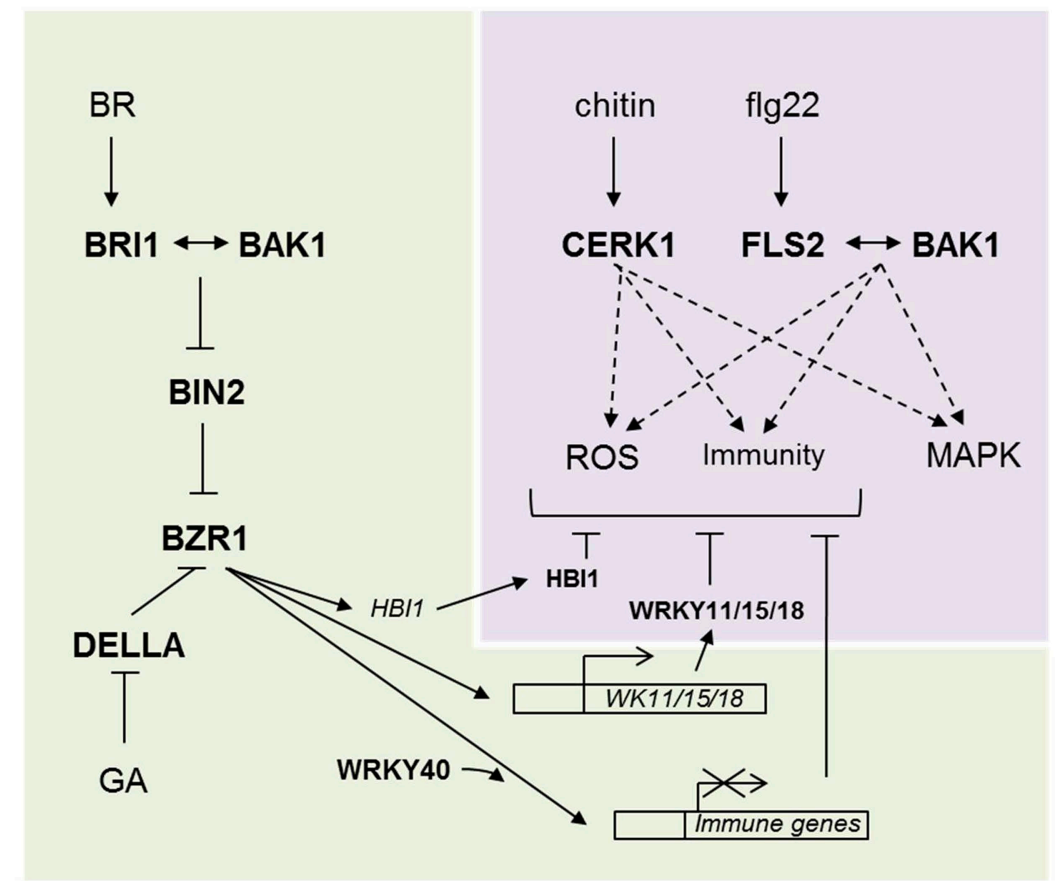

Figure 4. Activation of $B R$ signaling and BZR1 prioritizes growth over immunity in the dark. (A) and (B) Relative seedling growth inhibition of 10-day-old (A) Col-0, bri1-301 and bin2-1 or (B) Col-0 and BZR1 $\Delta$ seedlings induced by increasing concentrations of flg22 in either light or dark. (C) Relative seedling growth inhibition of 10-day-old Col-0 seedlings grown on medium supplemented or not with $B L(1 \mu M), G A(1 \mu M), B L+G A(1 \mu M+1 \mu M)$ or mock solution in light or dark. (D) Relative seedling growth inhibition of Col-0 or wrky40 seedlings induced by increasing concentrations of flg22 in either light or dark. Bars represent SE of $n=16$ (A, B and $\mathbf{D}$ ) or $n=8$ (C) Asterisks indicate a statistically significant difference compared to Col-0 in the same condition (light or dark and same concentration of flg22), according to a Student's t-test ( $p<0.05)$; 'a' indicates a statistically significant difference compared to the same genotype/ treatment and concentration of flg22 in light, according to a Student's $t$-test $(p<0.05)$. All experiments were repeated at least three times with similar results. Figure 4. Continued on next page 
Figure 4. Continued

Values are relative to Col-0 (A, B and D) or mock-treated seedlings (C) (set to 100). Absolute values of these experiments are shown in Figure 4-figure supplement 3. (E) Schematic model depicting the BZR1-mediated inhibition of PTI. Upon BR- and DELLA-dependent activation, BZR1 induces the expression of negative regulators of PTI, such as WRKY11, WRKY15, WRKY18, or HBI1. In addition, BZR1 also inhibits the expression of immune genes, acting cooperatively with WRKY40 and possibly other WRKYs. Ultimately, the BZR1-mediated changes in transcription would lead to the suppression of PTI signaling. The PTI signaling pathway is shadowed in violet; the BR signaling pathway is shadowed in green.

DOI: 10.7554/eLife.00983.018

The following figure supplements are available for figure 4:

Figure supplement 1. The BR-mediated suppression of seedling growth inhibition in the dark requires GA synthesis. DOI: 10.7554/eLife.00983.019

Figure supplement 2. Phenotype of the light- or dark-grown seedlings used in the seedling growth inhibition assays (Figure 4 and Figure 4-figure supplement 1).

DOI: 10.7554/eLife.00983.020

Figure supplement 3. Absolute fresh weight values of seedling growth inhibition assays.

DOI: 10.7554/eLife.00983.021

\section{ROS assays}

The measurement of ROS generation was performed as described in Albrecht et al. (2012). Leaf discs from five-week-old Arabidopsis plants were used in each experiment, as indicated in the figure legends. Total photon counts were measured over $40 \mathrm{~min}$ by using a high-resolution photon counting system (HRPCS218) (Photek, St Leonards on Sea, UK) coupled to an aspherical wide lens (Sigma Imaging, Welwyn Garden City, UK).

\section{Protein extraction and IP experiments}

Protein extraction and immunoprecipitation of Arabidopsis was performed as described in Schwessinger et al. (2011). Arabidopsis mesophyll protoplasts were prepared from 4 to 5-week-old plants, transfected with the indicated constructs and incubated for $16 \mathrm{hr}$ prior to BL treatment. Protein extraction of $N$. benthamiana was performed as described in Schwessinger et al. (2011); immunoprecipitations were performed using the $\mu$ MACS GFP Isolation Kit (Miltenyi Biotec, Church Lane Bisley, UK), following the manufacturer's instructions. In N. benthamiana, BZR1-GFP was expressed from the pUb-cYFP-Dest vector (Grefen et al., 2010); WRKY40-HA and WRKY6-HA were expressed from the PAM-PAT vector (AY436765; GeneBank). In protoplasts, WRKY40-HA was expressed from the pGWB414 vector (Nakagawa et al., 2007); the construct to express BZR1-GFP has been described elsewhere (Ryu et al., 2007). In both cases, samples were treated with $1 \mu \mathrm{M}$ epiBL solution for $1 \mathrm{hr}$ prior to protein extraction.

\section{MAP kinase activation assays}

MAP kinase activation assays were performed as described in Schwessinger et al. (2011). Phospho-p44/42 MAPK (Erk1/2; Thr202/Tyr204) rabbit monoclonal antibodies (Cell Signaling Technologies, Hitchin, UK) were used according to the manufacturer's protocol.

\section{RNA isolation and qPCR assays}

RNA isolation was performed from ten-day-old seedling following the protocol described in OnateSanchez and Vicente-Carbajosa (2008). First-strand cDNA synthesis was performed with the SuperScript III RNA transcriptase (Invitrogen, Paisley, UK) and oligo(dT) primer, according to the manufacturer's instructions. For qPCR reactions, the reaction mixture consisted of cDNA first-strand template, primers (5 nmol each) and SYBR Green JumpStart Taq ReadyMix (Sigma). qPCR was performed in a BioRad CFX96 real-time system. UBQ10 was used as the internal control; expression in mock-treated Col-0 seedlings was used as the calibrator, with the expression level set to one. Relative expression was determined using the comparative $\mathrm{Ct}$ method (2- $\Delta \Delta \mathrm{Ct}$ ). Each data point is the mean value of three experimental replicate determinations. Primers for At2g17740 are described in Albrecht et al. (2012); for NHL10 are described in Boudsocq et al. (2010); for LOX2 are described in Pandey et al. (2010); for UBQ10 (U-box) are described in Albrecht et al. (2012).

\section{Seedling growth inhibition assay}

Seedling growth inhibition assays were performed as described in Nekrasov et al. (2009). In brief, four-day-old Arabidopsis seedlings were grown in liquid Murashige-Skoog medium containing 1\% 
sucrose supplemented with flg22 and the appropriate chemicals. Seedlings were weighed between 6 and 10 days after treatment.

\section{Bacterial infections}

Induced resistance assays were performed as described previously (Zipfel et al., 2004). In brief, water or a $1 \mu \mathrm{M}$ flg22 solution were infiltrated with a needleless syringe into leaves of four-week-old Arabidopsis plants $24 \mathrm{hr}$ prior to bacterial inoculation (Pto DC $3000,10^{5} \mathrm{cfu} / \mathrm{ml}$ ). Bacterial growth was determined 2 days after inoculation by plating serial dilutions of leaf extracts.

Spray inoculation of $P$. syringae pv. cilantro ( $P$ ci) 0788-9 was performed as described in Schwessinger et al. (2011). In brief, bacteria were grown in an overnight culture in LB medium, cells were harvested by centrifugation, and pellets were re-suspended to OD600 $=0.02$ in $10 \mathrm{mM} \mathrm{MgCl}_{2}$ with $0.04 \%$ Silwet L-77. Bacterial suspensions were sprayed onto leaf surfaces and plants were kept uncovered. Bacterial growth was determined 3 days after inoculation by plating serial dilutions of leaf extracts.

\section{Meta-analysis}

Functional enrichment analyses of the Biological Process ontology were performed using VirtualPlant (Katari et al., 2010). Functional enrichment analysis of the Molecular Function ontology was performed using the Classification SuperViewer tool of the Bio-Array Resource for Arabidopsis Functional Genomics, BAR (Toufighi et al., 2005). Promoter analyses were performed using Athena (O'Connor et al., 2005).

\section{Acknowledgements}

RL-D is supported by a postdoctoral fellowship from Fundación Ramón Areces; APM is supported by a postdoctoral fellowship from the Federation of European Biochemical Societies. We thank Lena Stransfeld and the horticultural service at the John Innes Centre for excellent technical assistance, Yasuhiro Kadota for technical advice, and Christine Faulkner and all members of the Zipfel laboratory for fruitful discussions and helpful comments. We thank Zhiyong Wang, Joanne Chory, Ildoo Hwang, Dominique Roby, Eugenia Russinova, Xinnian Dong, Kang Chong, Zhiwiang Chen, Jun-Xian He, Jonathan Jones and David Guttman for sharing biological materials, and Sacco de Vries and Ben Scheres for excellent comments on the manuscript.

\section{Additional information}

Funding

\begin{tabular}{lll} 
Funder & $\begin{array}{l}\text { Grant reference } \\
\text { number }\end{array}$ & Author \\
\hline The Gatsby Charitable Foundation & & Cyril Zipfel \\
\hline $\begin{array}{l}\text { UK Biotechnology and Biological } \\
\text { Sciences Research Council }\end{array}$ & BB/G024936/1; & Cyril Zipfel \\
\hline Deutsche Forschungsgemeinschaft & SFB 670 & \\
\hline
\end{tabular}

The funders had no role in study design, data collection and interpretation, or the decision to submit the work for publication.

Author contributions

RL-D, Conception and design, Acquisition of data, Analysis and interpretation of data, Drafting or revising the article; APM, FB, CS, Conception and design, Acquisition of data, Analysis and interpretation of data; IES, Conception and design, Drafting or revising the article, Contributed unpublished essential data or reagents; CZ, Conception and design, Analysis and interpretation of data, Drafting or revising the article

\section{References}

Albrecht C, Boutrot F, Segonzac C, Schwessinger B, Gimenez-lbanez S, Chinchilla D, Rathjen JP, de Vries SC, Zipfel C. 2012. Brassinosteroids inhibit pathogen-associated molecular pattern-triggered immune signaling independent of the receptor kinase BAK1. Proceedings of the National Academy of Sciences of the United States of America 109:303-308. doi: 10.1073/pnas.1109921108. 
Alonso JM, Stepanova AN, Leisse TJ, Kim CJ, Chen H, Shinn P, Stevenson DK, Zimmerman J, Barajas P, Cheuk R, Gadrinab C, Heller C, Jeske A, Koesema E, Meyers CC, Parker H, Prednis L, Ansari Y, Choy N, Deen H, Geralt M, Hazari N, Hom E, Karnes M, Mulholland C, Ndubaku R, Schmidt I, Guzman P, Aguilar-Henonin L, Schmid M, Weigel D, Carter DE, Marchand T, Risseeuw E, Brogden D, Zeko A, Crosby WL, Berry CC, Ecker JR. 2003. Genomewide insertional mutagenesis of Arabidopsis thaliana. Science 301:653-657. doi: 10.1126/science.1086391.

Bai MY, Fan M, Oh E, Wang ZY. 2012a. A triple helix-loop-helix/basic helix-loop-helix cascade controls cell elongation downstream of multiple hormonal and environmental signaling pathways in Arabidopsis. Plant Cell 24:4917-4929. doi: 10.1105/tpc.112.105163.

Bai MY, Shang JX, Oh E, Fan M, Bai Y, Zentella R, Sun TP, Wang ZY. 2012b. Brassinosteroid, gibberellin and phytochrome impinge on a common transcription module in Arabidopsis. Nature Cell Biology 14:810-817. doi: $10.1038 /$ ncb2546.

Belkhadir Y, Jaillais Y, Epple P, Balsemao-Pires E, Dangl JL, Chory J. 2012. Brassinosteroids modulate the efficiency of plant immune responses to microbe-associated molecular patterns. Proceedings of the National Academy of Sciences of the United States of America 109:297-302. doi: 10.1073/pnas.1112840108.

Boudsocq M, Willmann MR, McCormack M, Lee H, Shan L, He P, Bush J, Cheng SH, Sheen J. 2010. Differential innate immune signalling via $\mathrm{Ca}(2+)$ sensor protein kinases. Nature 464:418-422. doi: 10.1038/nature08794.

Brotman Y, Landau U, Cuadros-Inostroza A, Tohge T, Fernie AR, Chet I, Viterbo A, Willmitzer L. 2013. Trichoderma-plant root colonization: escaping early plant defense responses and activation of the antioxidant machinery for saline stress tolerance. PLOS Pathogens 9:e1003221. doi: 10.1371/journal.ppat.1003221.

Casal JJ. 2013. Photoreceptor signaling networks in plant responses to shade. Annual Review of Plant Biology 64:403-427. doi: 10.1146/annurev-arplant-050312-120221.

Choudhary SP, Yu JQ, Yamaguchi-Shinozaki K, Shinozaki K, Tran LS. 2012. Benefits of brassinosteroid crosstalk. Trends in Plant Science 17:594-605. doi: 10.1016/j.tplants.2012.05.012.

De Rybel B, Audenaert D, Vert G, Rozhon W, Mayerhofer J, Peelman F, Coutuer S, Denayer T, Jansen L, Nguyen L, Vanhoutte I, Beemster GT, Vleminckx K, Jonak C, Chory J, Inzé D, Russinova E, Beeckman T. 2009. Chemical inhibition of a subset of Arabidopsis thaliana GSK3-like kinases activates brassinosteroid signaling. Chemical Biology 16:594-604. doi: 10.1016/j.chembiol.2009.04.008.

Dodds PN, Rathjen JP. 2010. Plant immunity: towards an integrated view of plant-pathogen interactions. Nature Reviews Genetics 11:539-548. doi: 10.1038/nrg2812.

Gallego-Bartolome J, Minguet EG, Grau-Enguix F, Abbas M, Locascio A, Thomas SG, Alabadi D, Blazquez MA. 2012. Molecular mechanism for the interaction between gibberellin and brassinosteroid signaling pathways in Arabidopsis. Proceedings of the National Academy of Sciences of the United States of America 109:13446-13451. doi: 10.1073/pnas.1119992109.

Gampala SS, Kim TW, He JX, Tang W, Deng Z, Bai MY, Guan S, Lalonde S, Sun Y, Gendron JM, Chen H, Shibagaki N, Ferl RJ, Ehrhardt D, Chong K, Burlingame AL, Wang ZY. 2007. An essential role for 14-3-3 proteins in brassinosteroid signal transduction in Arabidopsis. Developmental Cell 13:177-189. doi: 10.1016/j. devcel.2007.06.009.

Grefen C, Donald N, Hashimoto K, Kudla J, Schumacher K, Blatt MR. 2010. A ubiquitin-10 promoter-based vector set for fluorescent protein tagging facilitates temporal stability and native protein distribution in transient and stable expression studies. Plant Journal 64:355-365. doi: 10.1111/j.1365-313X.2010.04322.x.

Jaillais Y, Vert G. 2012. Brassinosteroids, gibberellins and light-mediated signalling are the three-way controls of plant sprouting. Nature Cell Biology 14:788-790. doi: 10.1038/ncb2551.

Journot-Catalino N, Somssich IE, Roby D, Kroj T. 2006. The transcription factors WRKY11 and WRKY17 act as negative regulators of basal resistance in Arabidopsis thaliana. Plant Cell 18:3289-3302. doi: 10.1105/ tpc.106.044149.

Katari MS, Nowicki SD, Aceituno FF, Nero D, Kelfer J, Thompson LP, Cabello JM, Davidson RS, Goldberg AP, Shasha DE, Coruzzi GM, Gutiérrez RA. 2010. VirtualPlant: a software platform to support systems biology research. Plant Physiology 152:500-515. doi: 10.1104/pp.109.147025.

Khan M, Rozhon W, Bigeard J, Pflieger D, Husar S, Pitzschke A, Teige M, Jonak C, Hirt H, Poppenberger B. 2013. Brassinosteroid-regulated GSK3/Shaggy-like kinases phosphorylate mitogen-activated protein (MAP) kinase kinases, which control stomata development in Arabidopsis thaliana. The Journal of Biological Chemistry 288:7519-7527. doi: 10.1074/jbc.M112.384453.

Kim TW, Michniewicz M, Bergmann DC, Wang ZY. 2012. Brassinosteroid regulates stomatal development by GSK3-mediated inhibition of a MAPK pathway. Nature 482:419-422. doi: 10.1038/nature10794.

Kim TW, Wang ZY. 2010. Brassinosteroid signal transduction from receptor kinases to transcription factors. Annual Review of Plant Biology 61:681-704. doi: 10.1146/annurev.arplant.043008.092057.

Lewis JD, Wu R, Guttman DS, Desveaux D. 2010. Allele-specific virulence attenuation of the Pseudomonas syringae HopZ1 a type III effector via the Arabidopsis ZAR1 resistance protein. PLOS Genetics 6:e1000894. doi: 10.1371/journal.pgen.1000894.

Li QF, Wang C, Jiang L, Li S, Sun SS, He JX. 2012. An interaction between BZR1 and DELLAs mediates direct signaling crosstalk between brassinosteroids and gibberellins in Arabidopsis. Science Signaling 5:ra72. doi: 10.1126/scisignal.2002908.

Monaghan J, Zipfel C. 2012. Plant pattern recognition receptor complexes at the plasma membrane. Current Opinion in Plant Biology 15:349-357. doi: 10.1016/j.pbi.2012.05.006.

Mukhtar MS, Deslandes L, Auriac MC, Marco Y, Somssich IE. 2008. The Arabidopsis transcription factor WRKY27 influences wilt disease symptom development caused by Ralstonia solanacearum. Plant Journal 56:935-947. doi: 10.1111/j.1365-313X.2008.03651.x. 
Nakagawa T, Suzuki T, Murata S, Nakamura S, Hino T, Maeo K, Tabata R, Kawai T, Tanaka K, Niwa Y, Watanabe Y, Nakamura K, Kimura T, Ishiguro S. 2007. Improved Gateway binary vectors: high-performance vectors for creation of fusion constructs in transgenic analysis of plants. Bioscience Biotechnology and Biochemistry 71:2095-2100. doi: 10.1271/bbb.70216.

Navarro L, Bari R, Achard P, Lison P, Nemri A, Harberd NP, Jones JD. 2008. DELLAs control plant immune responses by modulating the balance of jasmonic acid and salicylic acid signaling. Current Biology 18:650-655. doi: 10.1016/j.cub.2008.03.060.

Nekrasov V, Li J, Batoux M, Roux M, Chu ZH, Lacombe S, Rougon A, Bittel P, Kiss-Papp M, Chinchilla D, van Esse HP, Jorda L, Schwessinger B, Nicaise V, Thomma BP, Molina A, Jones JD, Zipfel C. 2009. Control of the pattern-recognition receptor EFR by an ER protein complex in plant immunity. The EMBO Journal 28:3428-3438. doi: 10.1038/emboj.2009.262.

Noguchi T, Fujioka S, Choe S, Takatsuto S, Yoshida S, Yuan H, Feldmann KA, Tax FE. 1999. Brassinosteroid-insensitive dwarf mutants of Arabidopsis accumulate brassinosteroids. Plant Physiology 121:743-752. doi: 10.1104/ pp.121.3.743.

O'Connor TR, Dyreson C, Wyrick JJ. 2005. Athena: a resource for rapid visualization and systematic analysis of Arabidopsis promoter sequences. Bioinformatics 21:4411-4413. doi: 10.1093/bioinformatics/bti714.

Oh E, Zhu JY, Wang ZY. 2012. Interaction between BZR1 and PIF4 integrates brassinosteroid and environmental responses. Nature Cell Biology 14:802-809. doi: 10.1038/ncb2545.

Onate-Sanchez L, Vicente-Carbajosa J. 2008. DNA-free RNA isolation protocols for Arabidopsis thaliana, including seeds and siliques. BMC Research Notes 1:93. doi: 10.1186/1756-0500-1-93.

Pandey SP, Roccaro M, Schon M, Logemann E, Somssich IE. 2010. Transcriptional reprogramming regulated by WRKY18 and WRKY40 facilitates powdery mildew infection of Arabidopsis. Plant Journal 64:912-923. doi: 10.1111/j.1365-313X.2010.04387.x.

Peng P, Yan Z, Zhu Y, Li J. 2008. Regulation of the Arabidopsis GSK3-like kinase BRASSINOSTEROID-INSENSITIVE 2 through proteasome-mediated protein degradation. Molecular Plant 1:338-346. doi: 10.1093/mp/ssn001.

Pieterse CM, Van der Does D, Zamioudis C, Leon-Reyes A, Van Wees SC. 2012. Hormonal modulation of plant immunity. Annu Review of Cell and Developmental Biology 28:489-521. doi: 10.1146/annurev-cellbio$092910-154055$.

Ranf S, Eschen-Lippold L, Pecher P, Lee J, Scheel D. 2011. Interplay between calcium signalling and early signalling elements during defense responses to microbe- or damage-associated molecular patterns. Plant Journal 68:100-113. doi: 10.1111/j.1365-313X.2011.04671.x.

Rushton PJ, Somssich IE, Ringler P, Shen QJ. 2010. WRKY transcription factors. Trends in Plant Science 15:247-258. doi: 10.1016/j.tplants.2010.02.006.

Ryu H, Kim K, Cho H, Park J, Choe S, Hwang I. 2007. Nucleocytoplasmic shuttling of BZR1 mediated by phosphorylation is essential in Arabidopsis brassinosteroid signaling. Plant Cell 19:2749-2762. doi: 10.1105/ tpc.107.053728.

Schon M, Toller A, Diezel C, Roth C, Westphal L, Wiermer M, Somssich IE. 2013. Analyses of wrky18 wrky40 plants reveal critical roles of SA/EDS1 signaling and indole-glucosinolate biosynthesis for Golovinomyces orontii resistance and a loss-of resistance towards Pseudomonas syringae pv. tomato AvrRPS4. Molecular Plant-Microbe Interactions 26:758-767. doi: 10.1094/MPMI-11-12-0265-R.

Schweizer F, Bodenhausen N, Lassueur S, Masclaux FG, Reymond P. 2013. Differential contribution of transcription factors to Arabidopsis thaliana defense against Spodoptera littoralis. Frontiers of Plant Science 4:13. doi: 10.3389/ fpls.2013.00013.

Schwessinger B, Roux M, Kadota Y, Ntoukakis V, Sklenar J, Jones A, Zipfel C. 2011. Phosphorylation-dependent differential regulation of plant growth, cell death, and innate immunity by the regulatory receptor-like kinase BAK1. PLOS Genetics 7:e1002046. doi: 10.1371/journal.pgen.1002046.

Shan L, He P, Li J, Heese A, Peck SC, Nurnberger T, Martin GB, Sheen J. 2008. Bacterial effectors target the common signaling partner BAK1 to disrupt multiple MAMP receptor-signaling complexes and impede plant immunity. Cell Host \& Microbe 4:17-27. doi: 10.1016/j.chom.2008.05.017.

Sun Y, Fan XY, Cao DM, Tang W, He K, Zhu JY, He JX, Bai MY, Zhu S, Oh E, Patil S, Kim TW, Ji H, Wong WH, Rhee SY, Wang ZY. 2010. Integration of brassinosteroid signal transduction with the transcription network for plant growth regulation in Arabidopsis. Developmental Cell 19:765-777. doi: 10.1016/j.devcel.2010.10.010.

Toufighi K, Brady SM, Austin R, Ly E, Provart NJ. 2005. The botany array resource: e-Northerns, expression angling, and promoter analyses. Plant Journal 43:153-163. doi: 10.1111/j.1365-313X.2005.02437.x.

Vert G, Chory J. 2006. Downstream nuclear events in brassinosteroid signalling. Nature 441:96-100. doi: 10.1038/nature04681.

Wang D, Amornsiripanitch N, Dong X. 2006. A genomic approach to identify regulatory nodes in the transcriptional network of systemic acquired resistance in plants. PLOS Pathogens 2:e123. doi: 10.1371/journal. ppat.0020123.

Wang ZY, Nakano T, Gendron J, He J, Chen M, Vafeados D, Yang Y, Fujioka S, Yoshida S, Asami T, Chory J. 2002. Nuclear-localized BZR1 mediates brassinosteroid-induced growth and feedback suppression of brassinosteroid biosynthesis. Developmental Cell 2:505-513. doi: 10.1016/S1534-5807(02)00153-3.

Wisman E, Cardon GH, Fransz P, Saedler H. 1998a. The behaviour of the autonomous maize transposable element En/Spm in Arabidopsis thaliana allows efficient mutagenesis. Plant Molecular Biology 37:989-999. doi: 10.1023/A:1006082009151.

Wisman E, Hartmann U, Sagasser M, Baumann E, Palme K, Hahlbrock K, Saedler H, Weisshaar B. 1998b. Knock-out mutants from an En-1 mutagenized Arabidopsis thaliana population generate phenylpropanoid 
biosynthesis phenotypes. Proceedings of the National Academy of Sciences of the United States of America 95:12432-12437. doi: 10.1073/pnas.95.21.12432.

Xu W, Huang J, Li B, Li J, Wang Y. 2008. Is kinase activity essential for biological functions of BRI1? Cell research 18:472-478. doi: 10.1038/cr.2008.36.

Xu X, Chen C, Fan B, Chen Z. 2006. Physical and functional interactions between pathogen-induced Arabidopsis WRKY18, WRKY40, and WRKY60 transcription factors. Plant Cell 18:1310-1326. doi: 10.1105/tpc.105.037523.

Yan Z, Zhao J, Peng P, Chihara RK, Li J. 2009. BIN2 functions redundantly with other Arabidopsis GSK3-like kinases to regulate brassinosteroid signaling. Plant Physiology 150:710-721. doi: 10.1104/pp.109.138099.

Yin Y, Wang ZY, Mora-Garcia S, Li J, Yoshida S, Asami T, Chory J. 2002. BES1 accumulates in the nucleus in response to brassinosteroids to regulate gene expression and promote stem elongation. Cell 109:181-191. doi: 10.1016/S0092-8674(02)00721-3.

Yu X, Li L, Zola J, Aluru M, Ye H, Foudree A, Guo H, Anderson S, Aluru S, Liu P, Rodermel S, Yin Y. 2011. A brassinosteroid transcriptional network revealed by genome-wide identification of BESI target genes in Arabidopsis thaliana. Plant Journal 65:634-646. doi: 10.1111/j.1365-313X.2010.04449.x.

Zhu JY, Sae-Seaw J, Wang ZY. 2013. Brassinosteroid signalling. Development 140:1615-1620. doi: 10.1242/ dev.060590.

Zipfel C, Robatzek S, Navarro L, Oakeley EJ, Jones JD, Felix G, Boller T. 2004. Bacterial disease resistance in Arabidopsis through flagellin perception. Nature 428:764-767. doi: 10.1038/nature02485. 